\title{
An Unusual Case of Early Onset Persistent Escherichia coli Septicemia Associated with Endocarditis
}

\author{
Sachin K. Gupta, MD ${ }^{1}$ Vishakha Nanda, MD ${ }^{1}$ Prashant Malviya, MD ${ }^{1} \quad$ Norman Jacobs, MD ${ }^{1}$ \\ Z. Naheed, MD $^{1}$ Tessy Joseph, MD ${ }^{1}$ \\ ${ }^{1}$ Department of Pediatrics, John H. Stroger, Jr. Cook County Hospital, \\ Chicago, Illinois \\ Address for correspondence Sachin Kumar Gupta, MD, 1901 W. Harrison \\ Street, Chicago, IL 60612 (e-mail: drsachin.apollo@gmail.com). \\ Am J Perinatol Rep 2013;3:105-106.
}
Abstract
Keywords
- Escherichia coli
- endocarditis
- early onset septicemia
- newborn
Escherichia coli infection is very common cause of early onset septicemia especially in very low-birth-weight newborns, but $E$. coli endocarditis has not been described in newborns. E. coli endocarditis, even in the adult population, is a rare and not well- characterized disease and is associated with high mortality. We report a very unusual presentation of persistent $E$. coli infection associated with endocarditis.

A term, male, small-for-gestational age infant was born to a 27-year-old Hispanic woman at 40 weeks' gestational age through normal spontaneous vaginal delivery. He received routine care after birth. Apgar scores were 9 and 9 at 1 and 5 minutes of age. The mother had an uneventful prenatal course, all prenatal laboratory values were unremarkable including group B streptococcal screen, and she had no maternal risk factor for infection.

The baby was transferred to the nursery in view of desaturations and oxygen requirement. On physical examination, he was noted to have dysmorphic facies (microcephaly, microstomia, micrognathia, and overriding sutures) and congenital anomalies including overlapping index finger on both hands, second and third toe syndactyly, and rocker bottom feet on both side. The anthropometric measurements were as follows: birth weight 2,560 $\mathrm{g}(<3$ rd percentile), head circumference $34.5 \mathrm{~cm}$ (10th to 25th percentile), and length $46.5 \mathrm{~cm}$ ( $<3$ rd percentile). The cardiovascular exam was remarkable for grade 2 systolic murmur. The respiratory, abdominal, and skin exam revealed normal findings. He was started on first-line antibiotics (ampicillin and gentamicin) on day 2 for persistent oxygen requirement, apnea, and abdominal distension, after evaluation for sepsis including blood and cerebrospinal fluid culture. The laboratory studies showed a white cell count of $23,600 / \mathrm{mm}^{3}$ with $59 \%$ neutrophils, $21 \%$ bands, $11 \%$ lymphocytes, hematocrit of $37 \%$, and platelets count of $144,000 / \mathrm{mm}^{3}$. The chest X-ray done showed normal cardiothymic silhouette with perihilar infiltrates on the left side.

Genetic karyotyping was obtained. The echocardiogram showed moderate-sized patent ductus arteriosus with left-toright shunt and two small ventricular septal defects in perimembranous and muscular septum. The head ultrasound was remarkable for increased echogenicity in the posterior fossa brain parenchyma, which showed improvement on later head ultrasound. The renal ultrasound was unremarkable. The blood culture was positive for Escherichia coli pan-sensitive to all antibiotics. Lumber puncture showed normal cell count and raised proteins $(259 \mathrm{mg} / \mathrm{dL})$. Initial urine culture showed no growth. Repeated blood cultures on days 5 and 7 again showed growth of pan-sensitive E. coli. A pediatric infectologist was consulted and antibiotics were changed. The wellplaced peripherally inserted central catheter (PICC) line (placed on day 4 in the right cephalic vein) was removed on day 7 because of concern it might be acting as a nidus for infection. The PICC line tip culture remained negative. The received

January 14, 2013 accepted after revision

May 22, 2013

published online

July 11, 2013
DOI http://dx.doi.org/ 10.1055/s-0033-1349346. ISSN 2157-6998.
Copyright (c 2013 by Thieme Medical Publishers, Inc., 333 Seventh Avenue, New York, NY 10001, USA. Tel: +1(212) 584-4662.
License terms

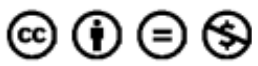




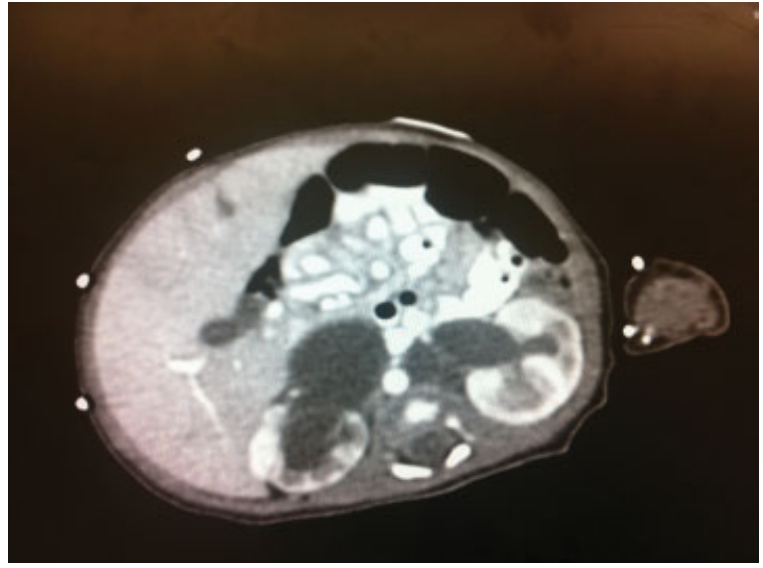

Fig. 1 Computed tomography scan of abdomen showing bilateral severe hydronephrosis, more on right side.

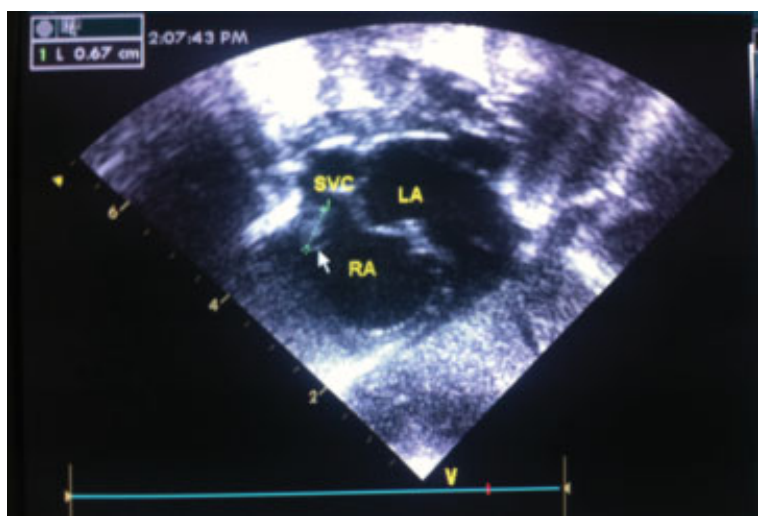

Fig. 2 Echocardiogram showing vegetation $(9 \times 3 \mathrm{~mm})$ at superior vena cava and right atrium junction. Abbreviations: LA, left atrium; RA, right atrium; SVC, superior vena cava.

patient was given six platelet transfusions during the first week for persistent thrombocytopenia. Repeat urine culture showed no growth. Total immunoglobulin levels were within normal limits, ruling out primary immunodeficiency disease.

Computed tomography scan of the abdomen was ordered to look for the focus of infection and was remarkable for bilateral severe hydroureteronephrosis, worse on the right, and bibasilar-dependent atelectasis (-Fig. 1). Repeat echocardiogram, to rule out endocarditis, was remarkable for vegetation $(9 \times 3 \mathrm{~mm})$ at the superior vena cava and right atrium junction (-Fig. 2). Antibiotics were continued for 28 days. The repeat blood cultures were negative. Karyotyping was positive for trisomy 18.

\section{Discussion}

The following features in our patient led us to the diagnosis of E. coli endocarditis: (1) persistent E. coli bacteremia; (2) echocardiographic evidence of vegetation; (3) the presence of risk factors for endocarditis-high-flow jet lesions (ventricular septal defect and patent ductus arteriosus), central venous catheter, and bilateral hydroureteronephrosis predisposing to urinary tract infection and endocarditis. Although both urine cultures were negative, they were tested after initiation of antibiotics.

E. coli endocarditis, a rare and poorly characterized disease even in the adult population, has not been described in the pediatric and neonatal age group. In adults, it has been mainly described in several small case series. ${ }^{1-5}$ The other risk factors associated with $E$. coli endocarditis in the adult population are injection drug use, diabetes, underlying heart disease, and prosthetic valves. ${ }^{1-5}$ The morality associated with $E$. coli is reported to be high, ranging from 17 to $53 \%$ in reported series. ${ }^{6,7}$

\section{Conclusion}

We describe the first case of neonatal $E$. coli endocarditis, a rare occurrence associated with high mortality (18 to 57\%). Our patient had trisomy 18 with associated congenital heart and kidney lesions, predisposing him to endocarditis. The high index of suspicion and appropriate selection of antibiotics for gram-negative bacteremia, bearing in mind the ampicillin-resistant E. coli, before culture and sensitivity report, and timely echocardiogram to rule out the rare occurrence of endocarditis in a persistently bacteremic newborn can significantly improve outcome in these infants.

\section{References}

1 Komshian SV, Tablan OC, Palutke W, Reyes MP. Characteristics of left-sided endocarditis due to Pseudomonas aeruginosa in the Detroit Medical Center. Rev Infect Dis 1990;12:693-702

2 Levine DP, Crane LR, Zervos MJ. Bacteremia in narcotic addicts at the Detroit Medical Center. II. Infectious endocarditis: a prospective comparative study. Rev Infect Dis 1986;8:374-396

3 Wieland M, Lederman MM, Kline-King C, et al. Left-sided endocarditis due to Pseudomonas aeruginosa. A report of 10 cases and review of the literature. Medicine (Baltimore) 1986;65:180-189

4 Mills J, Drew D. Serratia marcescens endocarditis: a regional illness associated with intravenous drug abuse. Ann Intern Med 1976;84: 29-35

5 Cooper R, Mills J. Serratia endocarditis. A follow-up report. Arch Intern Med 1980;140:199-202

6 Branger S, Casalta JP, Habib G, Collard F, Raoult D. Escherichia coli endocarditis: seven new cases in adults and review of the literature. Eur J Clin Microbiol Infect Dis 2005;24:537-541

7 Harries GE, Burtenshaw JML. Acute infective endocarditis due to Bacterium coli. Lancet 1937;2:803-804 